\title{
Simple kinetic information for transparent depth*
}

\author{
WILLIAM M. MACE $\dagger$ \\ Trinity College, Hartford, Connecticut 06106 \\ and \\ ROBERT SHAW \\ University of Minnesota, Minneapolis, Minnesota 55455
}

\begin{abstract}
The present paper reports three investigations of new kinetic information for transparent depth using computer-generated dot patterns. An initial demonstration showed that separation in depth could be obtained by translating rectangular lattices of dots through one another like intersecting columns of marching soldiers. The first two experiments showed that diagonal interactions between lattices created significantly stronger separation than did horizontal or vertical interactions (horizontal was, in turn, stronger than vertical) and that patterns which translated through one another without any of the individual elements intersecting were better separated than those whose rows or columns intersected in register. The third experiment showed that random patterns interacting in any direction created the strongest separations of all the patterns observed. Results were taken to indicate that a unified theory of depth information, developed in the context of James Gibson's ecological optics, must incorporate both spatial and kinetic structure in its specification of necessary and sufficient stimulus conditions.
\end{abstract}

What are the variables of optical stimulation that might specify the ordered layout of surfaces (including their depth relations) in the world for a human or animal observer? A corollary question is what are the minimal variables sufficient to do so? The first question has to do with the range of optical variables sufficient to specify depth to a human observer, while the latter question concerns the minimal set of variables of optical stimulation necessary to do so. Most studies have traditionally addressed themselves to the former question of empirically demonstrating that certain variables of optical information are merely sufficient and fail to address the issue of whether such variables are necessary if the relative separation of surfaces in depth is to be perceived at all.

The search for necessary conditions specifying depth is in keeping with the spirit of James Gibson's ecological optics (Gibson, 1966). Gibson maintains that visually perceiving stable properties of an environment is made possible by the existence of invariant relations in the light which are determined by invariant properties of the environment structuring that light. Where such correspondences hold, the structure of the light can be

\footnotetext{
*Based on a dissertation by the first author, submitted in partial fulfillment of the requirements for a $\mathrm{PhD}$ at the University of Minnesota. At the time, the author held an NIMH traineeship, MH06668, awarded through the Institute of Child Development. Other support was provided by a Career Development Award to Robert Shaw from the National Institute of Child Health and Human Development (1 K04-HD24010) and by grants to the University of Minnesota, Center for Research in Human Learning, from the National Science Foundation (GB-17590), the National Institute of Child Health and Human Development (HD-01136 and HD-0098), and the Graduate School of the University of Minnesota.

$\dagger$ Requests for reprints should be sent to Robert E. Shaw, Department of Psychology, University of Minnesota, Minneapolis, Minnesota 55455.
}

said to specify aspects of the environment and, hence, to contain information about it. The ecological optics strategy of investigation (logically prior to the development of a complete theory of perception) is devoted to experimentally identifying the optical structure specific to environmental features, structure which is invariant across common transformations of an ambient optic array. Its object, then, is to delimit the optical information available to any organism.

The first step in the strategy to unify depth information, and a major objective of this research project, is to find a visual display that intuitively seems to contain the least information which yields reliable depth perceptions. Having found such a demonstration, one could then analyze it experimentally in order to isolate the properties essential for depth and then be in a position to decide whether or not it suggests conditions common to other specifications of depth.

The most promising sources of minimal, but generalizable, cases are kinetic variables. Depth perceived in static patterns containing only one "cue" is typically ambiguous and unreliable; binocular depth perception depend's on the structure of organisms as well as on optic array variables. For instance, it is well known that a single perspective, as presented in a static projection of unfamiliar objects, geometrically specifies an indeterminately large family of patterns. Kinetic depth information, however, does not have either of these limitations. ${ }^{1}$ Furthermore, kinetic structure has greater ecological significance, since it applies to movements of organisms or motions of objects in an environment (more natural circumstances than stationary objects being received by immobile organisms).

Wallach and O'Connell (1953) demonstrated that shadows of rotating objects (e.g., solids, wire figures, and rods) provide sufficient optical information for the correct identification of their three-dimensional shape, 


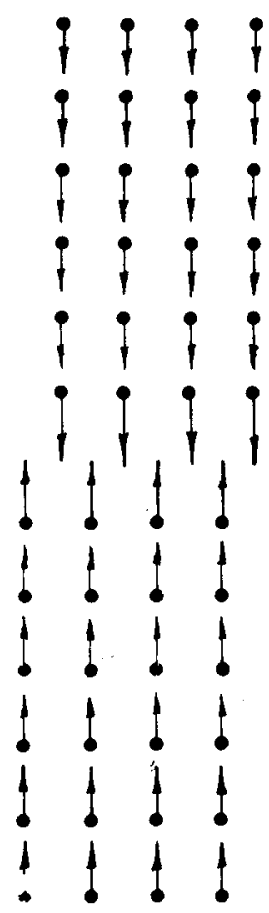

Fig. 1. Schematic representation of pattern used in pilot demonstration.

while shadows projected from the same objects when stationary do not. Wallach's claim that a change in at least two spatial dimensions is a necessary condition for the kinetic depth effect does not seem to be true, as Metzger's 1934 demonstration indicates (see White \& Mueser, 1960). What then is the minimal optical information for the kinetic depth effect?

In Metzger's display (shadows of revolving pegs), no kinetic perspective changes are available (and hence no changes in two spatial dimensions). However, there are harmonic velocity changes in the translatery motions of the shadows across the screen. But, since many other demonstrations exist which do not involve harmonic motion, this projective information cannot be considered essential to the specification of kinetic depth effects (Gibson, Gibson, Smith, \& Flock, 1959; Kaplan, 1969).

Gibson et al (1959) presented a display in which one set of small, amorphous shadows were translated rectilinearly through another set. Although no harmonic or perspective transformations were presented, Ss reported the appearance of one "surface" moving behind another. The motion parallax of the contra valent velocity vectors of the two sets of shadows was not sufficient, however, to provide a determinate depth effect (see Note 1). That is, one surface was seen as if through another (transparent depth effect), but neither set of shadows was seen consistently as being in front of the other (indeterminate direction of depth). Consequently, the authors concluded that motion parallax is not sufficient to account for the kinetic depth effect. Rather, they suggested that the effective optical information for kinetic depth was "topological breakage"-a change in the adjacent order of texture elements taken together with the invariant arrangement of each subset of elements. No further experiments were conducted to test their suggestion.

Subsequent research by Kaplan (1969) investigated a more specific type of topological breakage of optical texture which potentially includes the Metzger and Gibson et al cases, thereby emerging as an attractive candidate for being general as well as simple depth information. Kaplan showed that progressively adding (accretion) or subtracting (deletion) optical texture around texture that was not changed with respect to a point of observation specified a surface that was behind or occluded by the unchanged elements. This information, therefore, afforded the perception of determinately ordered depth at an occluding edge. It should be recalled that the shadow spots in both the Gibson et al and the Metzger displays crossed one another in many places. It is possible that these displays were also instances of Kaplan's accretion/deletion information for separation in depth where the transformation is not at a single margin but occurs in many microinstances as the shadows intersect. Whether or not the transformation was at a single margin perhaps could account for the difference between the transparent and opaque cases. This possibility was examined by constructing a display in which coherent patterns passed through one another without any elements intersecting.

\section{Demonstration}

The demonstration display, presented on the cathode ray tube of a laboratory computer, consisted of two rectangular lattices of points arranged in equally spaced parallel rows (or columns). One lattice moved from right to left, the other from left to right. The vertical displacement of each lattice relative to the other was half of the vertical distance between points so that the lattices intersected like rows of soldiers passing through one another. All four sides of each lattice were visible except when appearing or disappearing. Thus, the patterns as wholes intersected, while no single dots crossed over one another. Topological breakage was thereby defined, but accretion/deletion was not.

The result was striking and unambiguous. One lattice appeared to be in front of the other. All persons who saw the display immediately described it as one thing moving in front of another without any prompting. Either lattice could be seen in front or behind, thereby indicating that the direction of separation was indeterminate. However, it was extremely difficult to see the points as coplanar.

This demonstration rules out the hypothesis that the Gibson et al case was a species of occlusion. The 
following studies were conducted to begin to isolate the information that is effective.

\section{EXPERIMENT I THE EFFECTS OF FOUR POTENTIAL SEPARABILITY FACTORS}

\section{Method}

\section{Subjects}

Eleven adult Ss were selected from students and spouses of staff at the Institute of Child Development. Some were unfamiliar with perceptual experiments, others were themselves researcher experimenters, but none knew the purposes of this particular investigation.

\section{Apparatus and Physical Layout}

All patterns were computed on a PDP-12 computer and displayed on an auxiliary cathode ray tube (CRT), Display Type VR-12. The CRT was located next door to the computer in a dimly illuminated room, where the light was sufficient for the $\mathrm{E}$ to record comments and ratings.

A flat black piece of cardboard with a viewing hole cut out was used to cover the front of the CRT console. This hid a red indicator light on the front and eliminated extra leading or trailing margins which were visible in diagonally moving patterns that had margins. Reducing the size of the screen covered the patterns so that only one margin was visible at a time. The mask hole was egg-shaped and measured $17.78 \mathrm{~cm}$ vertically and $11.43 \mathrm{~cm}$ horizontally. This blocked out half of the horizontal display and none of the vertical. Since the $S$ was seated $91.44 \mathrm{~cm}$ from the screen, the corresponding visual angles were $11 \mathrm{deg} x$ 7 deg.

Two rectangular lattice patterns were defined in every display. Full lattices were composed of a $16 \times 16$ dot array. Half patterns, used in cases with margins, were $8 \times 16$ dots. Both the vertical and horizontal spacings of dots were defined as 32 scope units between adjacent dots. The resulting patterns were not square, however, because a horizontal unit on the CRT is $.0447 \mathrm{~cm}$, while a vertical unit is $.0323 \mathrm{~cm}$. Thus, the horizontal separation between lattice points that a $S$ sees is $1.42 \mathrm{~cm}$, or $54 \mathrm{~min}$ of arc, and the vertical separation is $1.04 \mathrm{~cm}$, or $36 \mathrm{~min}$ of arc.

All speeds were regulated by a programmable clock and were $25.6 \mathrm{msec} / \mathrm{scope}$ unit for motions in every direction. Thus, a point always moved from one lattice point to another in $819 \mathrm{msec}$. This means that the period of all motions was equal for every pattern, but that the absolute speeds for vertical, horizontal, and diagonal motions were different. In that order, the speeds were $1.2598 \mathrm{~cm} / \mathrm{sec}, \quad 1.7462 \mathrm{~cm} / \mathrm{sec}$, and $2.1526 \mathrm{~cm} / \mathrm{sec}$. Since absolute speed means little for perception, it was deemed better to keep the period rather than the speeds constant (Gibson, 1958).

\section{Stimulus Patterns}

Thirty-six patterns were compared for perceived separation in depth in Experiment I. The unifying condition was the same in all displays: two independent sets of rectangular lattice points each were translated identically. What varied were the separability conditions. Four types of optical discontinuity were selected for this initial investigation. Each pattern represented composition of variations on these factors in a completely crossed 2 by 2 by 3 by 3 experimental design. The factors and their levels are described below.

(1) Optical margin (present vs absent). It has already been shown that optical margins can be important for specifying occluding edges in the world (Kaplan, 1969). The demonstration described above also contained margins on all sides. In that case, when the patterns partially overlapped, there was a marked brightness difference between the overlapping and nonoverlapping portions of the display, which might have contributed to the separation in depth. Therefore, displays with a single margin were compared with displays without any margins to assess the role of this type of discontinuity in cases of transparent depth.

The contour used for vertical and horizontal cases was parallel to the direction of motion. That is, for vertical motion with contour, only the bottom half of the display contained a second lattice. In this way, the contour was present for the duration of a pattern's exposure. The contour on diagonal lattices, however, was not of this nature. Here, a moving lattice (the two non-back-and-forth cases) with contour eight columns wide was used. As these columns moved diagonally from lower left to upper right through the other pattern, a spatially vertical leading edge was defined. The eighth column defined a vertical trailing edge. New points were continuously produced at the bottom of the lattice so that there was no margin at the bottom. With the screen masked and the proper hole in the mask, the trailing edge disappeared from view just before a new leading edge appeared. Consequently, one, and only one, contour was always visible.

(2) Phase (in-phase vs out-of-phase). Since all patterns used were regular lattices and the spatial structure of each interacting lattice was identical, the term "phase" was used to indicate the spatial alignment of the intersecting patterns. Patterns like the demonstration in which the lattices intersected without elements crossing were called "out of phase." A display was "in phase" when its component lattices were aligned so that the rows or columns of each periodically overlapped perfectly. Phase was treated as a dichotomous variable to keep the total number of patterns manageable. Pilot demonstrations also suggested that it behaved more like an all-or-none factor than like a continuous one.

There are three major considerations for predicting the effect of phase. (a) The symmetry approach guiding much of this research would predict that the asymmetry of being out of phase should increase separability (see Shaw, McIntyre, \& Mace, in press). Following this approach, two collections of elements being out of phase in the plane would constitute a symmetry-breaking operation in the plane and thereby specify the noncoplanarity of the two collections. Therefore, if an effect of phase is found, then the symmetry analysis would provide at least one plausible account of what might constitute "topological breakage" in the plane. (b) Gibson et al's disruption of adjacent order would make the same prediction. More adjacent orders are disrupted in out-of-phase displays than in in-phase displays. (c) On the other hand, a Helmholtzian "unconscious inference" position should predict better separation for in-phase cases on the grounds that objects in the world must be separated in depth for their shadows to interact in such a way. Such an inference might follow from the knowledge that moving solid objects whose projected images (shadows) intersect must pass each other at different distances from the point of observation.

(3) Direction of motion (vertical vs horizontal vs diagonal). It was observed, in some demonstration patterns, that the direction one pattern moved across another might make a difference for perceived separation. Diagonal intersections appeared to achieve better separation than vertical or horizontal. Shaw, McIntyre, \& Mace (in press) have given a symmetry group analysis which motivates such a prediction. See Fig. 2 for selected patterns.

(4) Type of motion (one lattice was stationary and the other moving across it vs both lattices moving toward one another from opposite directions vs one lat tice oscillating back and forth within a stationary lattice). In the demonstration pattern, both lattices moved relative to the $O$. It was conceivable that having a 

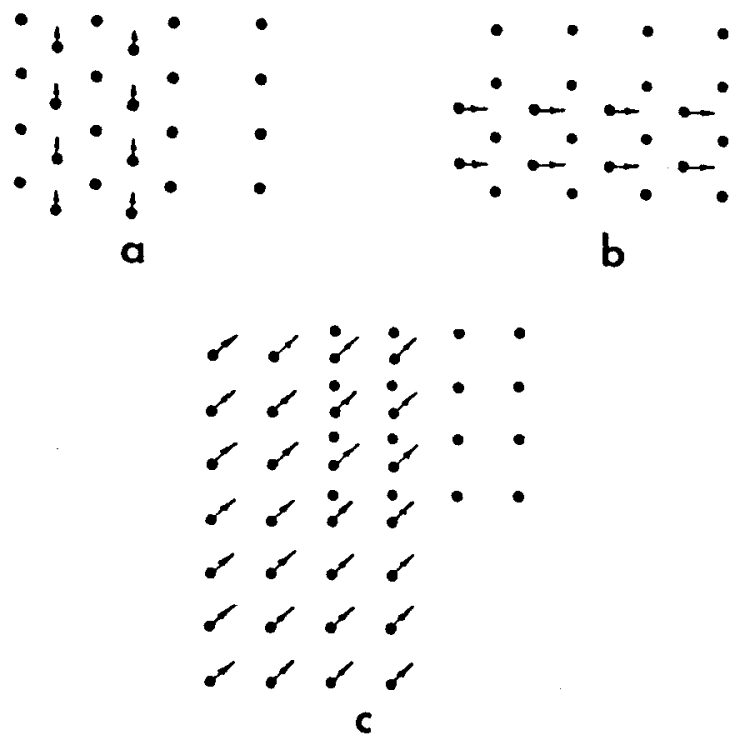

Fig. 2. Examples of patterns used in Experiment I. Vertical and horizontal dimensions are drawn to scale. All are out of phase with contour, having one lattice moving over a static lattice. Arrows represent direction of motion. (a) Vertical motion. (b) Horizontal motion. (c) Diagonal motion; unlike the vertical and horizontal cases, the lattice points do not renew continually at the edges of the screen; the total number of moving points is fixed so that leading and trailing margins are formed, and an $S$ sees only the area where the static points are.

more stable point of reference might affect separability. Consequently, displays where one lattice did not move were included.

The third motion condition was another test of Gibson et al's disruption-of-adjacent-order hypothesis. Imagine a stationary lattice with alternate rows or columns moving back and forth inside it with a period less than the distance between adjacent static points. If the motion vectors on the oscillating points were identical, then a coherent subset of points would have been defined. When these oscillating points were in-phase, no adjacent orders were disrupted. Could the separation nevertheless be in depth?

\section{Procedure}

An experimental session, lasting approximately $1 \mathrm{~h}$, was conducted with a single $S$ at a time. There were two parts to an experimental session. In the first part, the $S$ described each pattern in his own words. Each pattern was displayed until the $S$ felt he had nothing more to add to his description. When the $S$ was finished describing a display, the $\mathrm{E}$ changed the pattern with an appropriately programmed puh button plugged into an analogue channel of the computer.

The $E$ remained in the room with the $S$ throughout the experiment so that he could control the pattern presentations and record the S's responses. He sat behind the CRT, facing the $S$, so that he could not see the pattern on the screen but could see any gestures the $S$ used to elaborate his description of a pattern.

Each series of 36 patterns was presented in a different random order, computed at the beginning of a series. Hence, the $E$ never knew what pattern a $S$ was viewing except by the S's description.

At the end of the free description phase, a $S$ was allowed a short rest if he wanted it. The first part usually took about $45 \mathrm{~min}$. Once the spontaneous descriptions were completed, the E would not only have a good sample of a S's impressions, but the $S$ would be familiar with the patterns in preparation for the second part.

The second part of the experiment provided the primary analyzable data. The $S s$ were presented with a new randomization of the same set of patterns seen in the first part of the experiment. Ss were asked to rate the quality of the separation in depth achieved by each pattern on a 7-point scale (from $0=$ no separation to $6=$ strongest separation). For the few Ss who had made no spontaneous remarks about depth relations ( 3 of 31 in all three experiments), the instructions were modified to ask if indeed they had noticed separations in depth. Only one said that he had not. These three Ss were encouraged to look for depth separation in the patterns and instructed to proceed in this second part just as all of the other Ss had been instructed. For all Ss, the $\mathbf{E}$ rephrased the instructions whenever necessary to be sure they understood the task.

The rating phase ordinarily lasted less than $15 \mathrm{~min}$. A new pattern was displayed as soon as a $\mathrm{S}$ assigned a rating to the one being presented. The $\mathrm{E}$ recorded all of the ratings.

\section{Results}

\section{Ratings}

There was no obvious skewing in the distribution of ratings assigned by $\mathrm{Ss}$, and no significant correlation between cell means and variances. Consequently, it was legitimate to perform a four-factor within-Ss repeated measures analysis of variance on the raw ratings.

Because of a shortcoming in the randomization program, 1 of the 36 patterns was sometimes omitted and replaced by a display that had already been seen. This occurred for 8 of the 36 displays. Since each display constituted a cell in the analysis, it is equivalent to say that there was missing data in 8 of 36 cells. In 7 of them, only one observation was missing, while there were two missing in the other. The nine missing scores (out of a possible 396 observations) were filled in by randomly selecting a number between 0 and 6 for each one. The repeated pattern was scored on its first presentation only. The means for this analysis are presented in Table 1.

Two main effects stand out. (a) Out-of-phase > in-phase. The out-of-phase condition was judged consistently to be more firmly an instance of separation in depth than the in-phase condition $[F(1,10)=27.02$, $\mathrm{p}<.01]$. (b) Diagonal $>$ horizontal $>$ vertical. The direction of motion played a large role in perceived separability $[F(2,20)=50.29, p<.01]$. All pairwise differences were significant at the $p<.01$ level. Horizontal motion achieved greater separability than the vertical motion [.99 Scheffé confidence interval (CI) on the difference between horizontal and vertical was $1.45 \pm .83$ ], and diagonal motion had a greater effect than horizontal (Scheffé $.99 \mathrm{CI}=.96 \pm .83$ ). The difference between vertical and diagonal holds a fortiori. Thus, vertical, horizontal, and diagonal motions were increasingly effective, in that order.

There were two significant interactions involving significant main effects, but neither interaction obviates the meaning of the main effects. First there was a Margin 
by Phase interaction $[F(1,10)=9.25, p<.05]$, which indicated that the presence of a margin was significant only when patterns were in-phase. The Scheffé $.95 \mathrm{CI}$ for the difference between margin and no margin for in-phase patterns was $.83 \pm .66$, which does not cover zero. The same difference in out-of-phase patterns does cover zero (Scheffé .95 CI $=.4 \pm .66$ ), but was in the same direction. Another way to describe the interaction is to say that phase mattered only when there was no margin (Scheffé $.99 \mathrm{CI}$ on out-of-phase minus in-phase at no margin $=1.17 \pm .56$ ). Again, the main effect of phase should not be disregarded because of this interaction. Out-of-phase patterns consistently were judged as having more separation than their in-phase counterparts. There were 18 direct comparisons of this sort in the experiment, and the out-of-phase member received a higher mean rating than the in-phase member in every case but one.

The Phase by Direction interaction was significant. An out-of-phase pattern yielded greater depth than an in-phase pattern when the direction of motion was vertical (Scheffé .95 CI $=1.22 \pm .76$ ) or horizontal (Scheffé $.95 \mathrm{CI}=.80 \pm .76$ ), but not when the motion was diagonal. Thus, being out-of-phase did not contribute further to separability achieved by diagonal motion. The main effect for direction remained important. There were 12 cell comparisons between vertical and horizontal and between horizontal and vertical. All 12 pairwise comparisons in each case were in the direction of the main effect.

It should be noted that for displays without margins where the motion was in-phase and vertical, seven Ss called the separation 0 (flat) in the case where one lattice moved continuously over a static lattice, and six gave a rating of 0 to the case where both lattices moved through one another. All but one of the remaining ratings of these two patterns were $1 \mathrm{~s}$.

\section{Spontaneous Verbal Reports}

It would not be meaningful to report formal analysis of measures based on the comments of Ss, since so many crucial but uncontrolled variables contribute to such a description. Because Ss were not instructed to mention depth or any other special characteristic of a perceived pattern, a person sometimes perceived a separation in depth without reporting it-as evidenced by comments made after the instructions for the second part, stressing depth, were given. Despite these qualifications, it is interesting to note that the proportional distribution of depth responses on each pattern very closely reflected the pattern of mean ratings of strength of separability collected in the second part of the experiment (see Mace, 1971).

The depth achieved was indeterminate. No variable seemed to specify which pattern was in front or behind. For each of the continuously moving patterns (where one moved continuously over a stationary pattern, or
Table 1

Mean Ratings for Quality of Separation in Depth in Experiment I

\begin{tabular}{lccccc}
\hline & \multicolumn{2}{c}{ Contour } & & \multicolumn{2}{c}{ No Contour } \\
\cline { 2 - 3 } \cline { 5 - 6 } \multicolumn{1}{c}{ Type of Motion } & $\begin{array}{c}\text { In } \\
\text { Phase }\end{array}$ & $\begin{array}{c}\text { Out of } \\
\text { Phase }\end{array}$ & $\begin{array}{c}\text { In } \\
\text { Phase }\end{array}$ & $\begin{array}{c}\text { Out of } \\
\text { Phase }\end{array}$ \\
\hline & \multicolumn{4}{c}{ Vertical } \\
One Moves, One Static & 1.36 & 2.18 & .45 & 2.64 \\
Both Move & 1.45 & 2.64 & .54 & 2.36 \\
Back and Forth & 1.63 & 2.36 & 1.09 & 1.73 \\
& \multicolumn{4}{c}{ Horizontal } \\
One Moves, One Static & 2.82 & 2.91 & 2.27 & 3.73 \\
Both Move & 3.73 & 4.09 & 2.64 & 4.00 \\
Back and Forth & 2.73 & 3.45 & 2.36 & 3.18 \\
& \multicolumn{4}{c}{ Diagonal } \\
One Moves, One Static & 4.45 & 3.73 & 3.27 & 4.36 \\
Both Move & 4.82 & 5.00 & 3.82 & 4.18 \\
Back and Forth & 4.18 & 4.27 & 3.27 & 4.09 \\
\hline
\end{tabular}

Note $-N=11$

where both moved), at least one $S$ found it to reverse in depth spontaneously. There were three patterns which at least half of the Ss saw reverse. These were all cases where there was no margin and both lattices were moving. The fact that the other patterns did not reverse much or reverse at all does not mean that depth was determinately ordered. There was never unanimous agreement across Ss on which lattice was in front or behind. Where one pattern moved over a static pattern, the moving one was most often seen in front (which might be attributed to a biasing of perceptual decisions toward motion parallax).

Perhaps the feature of Ss' descriptions which is most convincing as evidence for the immediacy of the depth perception was the occasional use of depth as the basic descriptive anchor. One S, reporting on a display with a margin formed by a half-matrix moving left to right in phase over the lower portion of a full stationary lattice said, "The pattern on top is moving left to right." He did not say that the dots moving left to right on the lower half of the screen appeared to be on top. Another S, observing a full lattice moving in phase over a stationary full lattice reported, "There are two surfaces. The one in back is moving to the right and stopping. They reverse motions. Then the one in front moves to the right." Thus, a reversal in depth was taken as a reversal in motion of patterns that did not shift in depth. Five different Ss described at least one pattern with the depth relation as anchor.

Depth judgments did not depend on Ss' organizing identically moving points as a surface. Columns or rows were often seen as strings of beads, swimmers in lanes, traffic lanes, etc., and still judged to be on a different plane from the differently moving points. ${ }^{2}$

\section{Discussion}

The results showed that perceived separation in depth could be manipulated from near flatness to good 


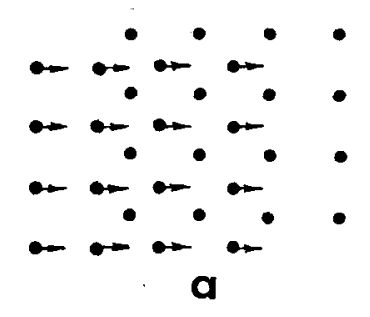

vertical motion, however, was unexpected. An attempt to replicate this finding is reported in the next study.

The weakness of the margin factor was mildly surprising. It is conceivable that the presence of a margin would have been more effective had a leading and trailing contour, rather than a lateral one, been used. This possibility is also reported in the next experiment.

\section{EXPERIMENT II THE EFFECTS OF CONTOUR AND DIRECTION OF MOTION}

New horizontal and vertical patterns, which had leading and trailing margins, were constructed for comparison with similar patterns from Experiment I, which had either lateral margins or no margins. Both in-phase and out-of-phase patterns were used to see if the interactions as well as the main effects of Experiment I were replicable. The difference between horizontal and vertical intersections could also be tested again. Diagonal patterns were not included on the grounds that their effectiveness was established.

\section{Method}

Fig. 3. Examples of patterns used in Experiment II. Leading and trailing margins are formed by fixing the number of moving points as in Fig. 2c. (a) Horizontal. (b) Vertical.

separation without any accretion/deletion of texture or any other previously reported depth "cue." Continuously moving lattices which moved vertically in phase and had no margins were rated by more than half of the Ss as having no depth separation at all. There was a slight bias in favor of Ss reporting greater apparent depth for lattices moving horizontally. There was also a bias in favor of seeing the faster lattice as being in front. Both of these effects might be due to a motion parallax effect, since the horizontally moving lattice has a slightly greater rate of movement across the cathode ray tube than a vertically moving one. It should, however, be noted, as stated earlier, that parallactic motion has not proven to be a reliable cue for depth (Gibson et al, 1959). Also, the fact that motion parallax to some extent contributes a bias to the perceived relative ordering of surfaces in depth is not evidence that it qualifies as the minimally sufficient information for separation being sought. Consequently, mere relative motion and the disruption of adjacent orders of elements are each insufficient to produce depth. The law which specifies depth information will definitely have to relate both spatial and kinetic organization.

Two factors, direction and phase, clearly contributed to perceived separability; one, margin, was less important, and the fourth, type of motion, was of no consequence.

Diagonal intersections and being out-of-phase were expected to increase separability on the basis of prior observations. The advantage of horizontal motion over

\section{Subjects}

Ten new Ss were selected in the same way as in Experiment I.

\section{Apparatus}

A different mask was used for the CRT in order to guarantee that only one margin was visible at any time where leading and trailing margins were present in displays moving vertically or horizontally. The hole in this mask was slightly longer on the horizontal than on the vertical dimension. Its vertical axis measured $8.89 \mathrm{~cm}$ and its horizontal axis was $11.13 \mathrm{~cm}$. The corresponding visual angles were approximately 5.5 and $7 \mathrm{deg}$, respectively. Roughly half of the screen was covered in each direction.

\section{Stimulus Patterns}

(1) Margins (new vs old vs none). Here "new" refers to horizontal and vertical patterns with leading and trailing margins. In the horizontal case, for example, a single cycle consisted of 16 columns of 16 spots moving serially into the field of view, all being simultaneously visible when they covered the other pattern (which filled the display field), then serially disappearing. The other two conditions are the same as in the previous study.

(2) Phase (in-phase vs out-of-phase). Same as Experiment I.

(3) Direction (vertical vs horizontal). Same as Experiment I.

For all displays, a static lattice of dots filled the screen. The second lattice moved continuously across the first. Thus, there were only four new patterns-those with new contour combined with the two phases and two directions. See Fig. 3 for selected examples.

\section{Procedure}

The entire procedure was identical to Experiment I. Less than $30 \mathrm{~min}$ was required to complete a session. 


\section{Results}

The means from Experiment II are shown in Table 2. A three-factor within-Ss repeated measures analysis of variance was performed. There were two significant main effects. Once again, out-of-phase patterns were judged to be separated better than in-phase patterns $[F(1,9)=$ $5.16, \mathrm{p}<.05]$ and horizontally moving patterns were separated better than vertical patterns $[F(1,9)=18.86$, $\mathrm{p}<.01]$. The new contour cases received higher ratings than the old lateral margins, which in turn received higher ratings than no contour at all. But this was only a trend. The overall effect was not at all significant. Finally, there were no significant interactions. Out-of-phase patterns were perceived as having more depth than in-phase patterns in every direct comparison this time. The verbal reports collected were very similar to those in the previous study.

\section{Discussion}

It is clear that the presence of any margin, new or old, played a subordinate role to phase and direction of motion in separating lattices in depth. The margin factor contributed neither a main effect nor an interaction in this study.

The difference between horizontal and vertical moving patterns, as well as the phase difference, remained consistent with Experiment $I$. This effect is investigated further in the next study.

\section{EXPERIMENT III EFFECTIVE FRAMES OF REFERENCE}

In Experiment I, it was found that horizontal motion achieved greater depth than vertical motion and that diagonal motion produced more effective depth than horizontal motion. Experiment II confirmed that horizontal motion gave better depth than vertical motion. What reference system is most important for defining these directions? There are three available systems of reference: the geographical, the self, and the internal structure of the pattern. In the geographical environment, vertical is defined by gravity and horizontal by the ground plane. In a self-reference system (visual), vertical is defined from head to toe, or, perhaps, from hair to chin, and horizontal is defined from ear to ear. In the pattern itself, the rows and columns of the dot matrices are perpendicular to one another, so that they define an internal diagonal independent of the orientation of the display in either the geographical or self-reference system (Pufall \& Shaw, 1973).

The patterns of Experiment III were constructed to compare systematically the effectiveness of these reference systems for separating in depth. Although run as a single session for each $\mathrm{S}$, the study was conceptualized as two experiments. The first involved
Table 2

Mean Ratings for Quality of Separation in Depth in Experiment II

\begin{tabular}{|c|c|c|c|c|c|c|}
\hline \multirow[b]{2}{*}{$\begin{array}{l}\text { Direction } \\
\text { of Motion }\end{array}$} & \multicolumn{2}{|c|}{ Old Contour } & \multicolumn{2}{|c|}{ New Contour } & \multicolumn{2}{|c|}{ No Contour } \\
\hline & $\begin{array}{c}\text { In } \\
\text { Phase }\end{array}$ & $\begin{array}{l}\text { Out of } \\
\text { Phase }\end{array}$ & $\begin{array}{c}\text { In } \\
\text { Phase }\end{array}$ & $\begin{array}{l}\text { Out of } \\
\text { Phase }\end{array}$ & $\begin{array}{c}\text { In } \\
\text { Phase }\end{array}$ & $\begin{array}{l}\text { Out of } \\
\text { Phase }\end{array}$ \\
\hline Vertical & 1.8 & 2.4 & 2.2 & 2.7 & .7 & 1.7 \\
\hline Horizontal & 2.7 & 3.9 & 2.9 & 4.0 & 2.3 & 3.2 \\
\hline
\end{tabular}

Note $-N=10$

the relation between diagonal and vertical, the two extremes of effectiveness, while the second involved the vertical-horizontal comparison.

\section{Part I \\ The Relation Between Diagonal and Vertical}

Ss viewed a lattice moving along the geographical vertical over a static matrix of dots in either an upright position parallel to the display or leaning on a headrest at $45 \mathrm{deg}$ to it. They also viewed a geographically oriented, diagonally moving lattice that moved over the static set of dots in either the upright or 45-deg tilted position. These displays exhaust all possible combinations of vertical and diagonal defined in each frame of reference. If the effective diagonal and vertical were defined in a self-reference system, the effects should be weakest whenever the S's orientation is parallel to the axis of motion in the display. If the geographic reference system were dominant, the vertical defined in the display would be weakest regardless of head position.

In these four patterns, the reference system defined by the pattern itself is redundant with the geographical reference system and, hence, indistinguishable from it. To separate out this factor, a random pattern was employed in the same four conditions as the rectangular lattice. Notice that in a random pattern no internal diagonal can be defined. If either of the other two reference systems acted to increase the separation of this pattern, that would argue strongly for the general efficacy of that system of reference. If the random pattern were not affected by a gravitational or self-reference system's diagonal, that would establish the pattern configuration itself as the major variable-in the absence of other complicating factors.

\section{Part II \\ The Reference Systems Defining Horizontal and Vertical}

The second part of the experiment involved the vertical-horizontal comparison. Ss observed a geographically horizontal moving lattice with their heads in either the upright position or lying down at 90 deg on a headrest. For the same reasons given for the diagonal-vertical comparison, mutatis mutandis, this 
Table 3

Mean Ratings for Quality of Separation in Depth in Experiment III

\begin{tabular}{cccc}
\hline $\begin{array}{c}\text { Geographical } \\
\text { Reference } \\
\text { System }\end{array}$ & $\begin{array}{c}\text { Self- } \\
\text { Reference } \\
\text { System }\end{array}$ & $\begin{array}{c}\text { Random } \\
\text { Pattern }\end{array}$ & Lattice \\
\hline \multirow{2}{*}{ Diagonal } & Diagonal & 4.6 & 3.3 \\
& Vertical & 4.3 & 2.6 \\
Vertical & Diagonal & 3.6 & .8 \\
& Vertical & 4.3 & .5 \\
\hline
\end{tabular}

Note $-N=10$

could separate out the effects of the geographical vs self-reference systems, with particular interest focused on the vertical-horizontal effects noticed in the previous studies.

\section{Method}

Ten new Ss were selected as before. Several details changed, however, as a consequence of using a headrest.

The headrest itself was a padded piece of plywood hinged to another piece of plywood. These two pieces could either lie flush with one another or be wedged in a fixed position of $45 \mathrm{deg}$. The two boards constituting the headrest were mounted on an adjustable stand so that each $S$ could choose the most comfortable height. The headrest was placed on the right of every $\mathbf{S}$.

\section{Displays}

Twelve displays were used, eight belonging to Part I of the experiment and four belonging to Part II. The first eight were composed of all combinations of the following factors: (1) head upright vs head at $45 \mathrm{deg}$; (2) pattern moving vertically vs pattern moving diagonally; and (3) random pattern vs lattice pattern. One can see that there were really only four different patterns. It is by counting the two head positions as part of a pattern that eight displays were defined (i.e., 2 head positions by 2 directions of motion by 2 types of pattern $=8$ ).

The program which presented the displays employed a pseudorandom number generator to select the points for the random patterns. The static and moving patterns were identical. Since these points were selected whenever the program was loaded, and the program was loaded four different times in the course of the experiment, four different random patterns were used.

All displays were composed of one stationary pattern and one moving across it continuously. None had margins and all the lattices were spatially out-of-phase.

Part II consisted of four patterns made by combining one vertically moving lattice display and one horizontal display with upright and 90-deg (with respect to the geographical vertical) head positions. The display, which was moving along the geographical vertical and viewed from an upright head position, was identical to a pattern in the first set of eight. These were treated as separate patterns in the execution and analysis of the experiments; that is, the same pattern actually occurred twice in the set of 12 . However, since all 12 were presented in random order, there was no systematic bias to determine whether the first or second presentation of this pattern belonged to the first or second part of the experiment.

\section{Procedure}

Unlike in the previous two experiments, the $\mathrm{E}$ sat behind the Ss, where he could see the CRT display. This was necessary so that he could adjust the headrest to the appropriate position before presentation of each pattern. It was felt that the phenomena were sufficiently well established to discount the possibility of an E's knowledge of the pattern affecting the results.

The instructions and procedure were similar to those in Experiments I and II, with the addition of the descriptions of the use of the headrest. The patterns belonging to both parts of the experiment were presented randomly. In order to place the headrest appropriately, the E had a copy of the predetermined random order so that he could inform the $S$ of the orientation and have the $\mathrm{S}$ assume the proper position before each pattern came on. The $S$ remained in this position until he was finished describing the pattern and rating its depth.

\section{Results}

The means for Part I of Experiment III appear in Table 3. The eight patterns of Part I were analyzed in a three-factor within-Ss repeated measures analysis of variance. There were two significant main effects and no significant interactions. By far the strongest effect was the depth difference between the random and lattice patterns. The random pattern achieved far better separation than did the lattice $[F(1,9)=28.56$, $\mathrm{p}<.01]$. Geographical orientation was also significant. Patterns moving diagonally to gravity were rated as having better separation in depth than vertical patterns $[F(1,9)=6.74, p<.05]$.

Scheffé CIs on contrast between the geographical vertical and horizontal within each pattern showed that the main effect was carried by the lattice patterns. The difference between the diagonal and vertical means is not significant for the random pattern (Scheffé $.95 \mathrm{CI}=$ $.5 \pm 1.04$ ), but is significant for thelattices (Scheffé .95 $\mathrm{CI}=2.3 \pm 1.04$ ). This differential effect of the geographical frame of reference on the two patterns was reflected in the marginal interaction of those two factors $[F(1,9)=3.37, p<.10]$.

In Part II, there were no significant differences whatsoever.

\section{Spontaneous Descriptions}

The reports of depth in random patterns were stronger than in any other pattern previously shown. Rather than just saying that one set of dots looked closer than another set, Ss occasionally said things like, "I have a feeling that there is depth in the pattern." Ss" spatial organization varied widely, as usual. The random patterns were seen at one time or another as bubbles, clouds, balloons, two planes, two pieces of transparent plastic, stars, and stars with identifiable constellations. Again, none of these had any bearing on whether or not a $\mathrm{S}$ reported depth.

\section{Discussion}

It is clear that the rating scale values Ss selected to characterize the separability of any particular pattern must be a function of the whole set. In this last study, where six patterns produced very strong depth effects 
(four were random and two were diagonally moving lattices), the geographically horizontal pattern viewed with head upright could muster a mean of only 1.2. Seemingly, the contrast of the Part II patterns with those several strong patterns was sufficient to obliterate whatever distinctions could have been made among the displays of Part II. ${ }^{3}$

Turning to the reference systems, the self-reference system was irrelevant. Geographical orientation did make a significant difference in the case of the lattice patterns. However, as noted above, the geographical and internal frames of reference were confounded in the lattices. The fact that the random patterns achieved strong depth in every direction of motion and were not significantly affected by motions relative to either the geographical or personal reference system supports the claim that separability motions must be defined with respect to the patterns involved and do not relate in any direct way to some other reference system.

\section{CONCLUSION}

If it is true, as supposed necessary in the introduction, that certain abstract conditions hold for every case of separation in depth, then the displays reported here may be particularly useful-for they appear to contain both simplicity and complexity in sufficient measure to qualify as a key empirical step in the analysis of depth separation. On the side of simplicity is the fact that only linear translatory motion of easily described patterns through one another is required for separation. This seems intuitively less complicated than previously formulated situations containing depth information (though, fortunately, little of ultimate consequence rides on whether or not such strategic intuitions of simplicity are widely agreed to). On the side of complexity is the fact that the effective variables cannot be defined as locally as the accretion/deletion of texture or the simple disruption of adjacent orders of units. The function of disruption must, of course, continue to be performed in our future comprehensive theory of depth separation, but the disruption depends on an accompanying conception of coherence to be disrupted. The fact that depth information was virtually absent in the patterns whose motion was in-phase and vertical and very much present in the diagonally moving and random displays indicates that the minimal description of coherence over which disruption relevant to depth will be defined must contain more structure than merely adjacent orders of texture units which may be disturbed by interruption or disappearance.

One approach that promises to be helpful in these matters is that of symmetry group theory. It seems to have the promise of simplifying unification consistent with the main thrusts of Gibson's ecological optics. Shaw, McIntyre, and Mace (in press) have taken the first steps toward elaborating this approach by applying it to the phenomena reported in this paper as well as to the specification of solid shape and aging of human faces. A description of these theoretical beginnings extends far beyond the scope of this report of the data, but it is important to indicate that, wisely or foolishly, this work is being developed in a context other than stringing another bead on psychology's necklace of effects.

\section{REFERENCES}

Gibson, E. J., Gibson, J. J., Smith, O. W., \& Flock, H. R. Motion parallax as a determinant of perceived depth. Journal of Experimental Psychology, 1959, 58, 40-51.

Gibson, J. J. Research on the visual perception of motion and change. Paper read at 2nd Symposium on Physiological Psychology, Office of Naval Research, 1958. Reprints in I. M. Spigel (Ed.), Readings in the study of visually perceived movement. New York: Harper \& Row, 1965.

Gibson, J. J. The senses considered as perceptual systems. Boston: Houghton-Mifflin, 1966.

Kaplan, G. Kinetic disruption of optical texture: The perception of depth at an edge. Perception \& Psychophysics, 1969, 6, 193-198.

Mace, W. An investigation of spatial and kinetic information for separation in depth using computer generated dot patterns. Unpublished doctoral dissertation, University of Minnesota, 1971.

Pufall, P., \& Shaw, R. E. An analysis of the development of chilren's spatial reference systems. Cognitive Psychology, 1973 , in press.

Shaw, R. E., McIntyre, M., \& Mace, W. The role of symmetry in event perception. In H. Pick and R. MacLeod (Eds.), Studies in perception: Essays in honor of J. J. Gibson. Ithaca, N.Y: Cornell University Press, in press.

Wallach, H., \& O'Connell, D. The kinetic depth effect. Journal of Experimental Psychology, 1953, 45, 205-217.

White, B. W., \& Meuser, G. Accuracy in reconstructing the arrangement of elements generating kinetic depth displays. Journal of Experimental Psychology, 1960, 60, 1-11.

\section{NOTES}

1. It should be noted, however, that kinetic depth information is not necessarily unambiguous in all cases. For instance, although the depth separation of intersecting moving surfaces is nearly always specified, the order of the surfaces relative to the $O$ might not be if the surfaces are partially transparent.

2. An unexpected and fascinating motion effect occurred in in-phase patterns. Each time the two component lattices of a display were congruent, the movement appeared to slow down, even though, in reality, it was linearly constant. Ss described this nonlinear motion as being jerky. This effect is described more fully in the body and appendix of Mace (1971).

3. Recall that all of the lattices used in these experiments were rectangular, i.e., the vertical and horizontal spacing between points was not equal. A recent full replication of Experiment I (10 Ss), using square lattices, showed that only the diagonal interaction significantly accounted for perceived differences in quality of separation. However, the horizontal patterns were still stronger than the vertical ones, and this difference almost reached statistical significance. Hence, the horizontal-vertical difference is probably not attributable solely to the rectangularity of the lattices in in the experiment being described. The square array experiment failed to reveal any phase effect. The trend in favor of out-of-phase patterns was very small. Thus, diagonal interaction with respect to internal pattern axes continued to emerge as the major factor contributing to separability.

(Received for publication December 18, 1972; revision received September 25,1973 .) 